\title{
Study of Writing Methodologies for Graphical Display on Flat Cathode Ray Tube
}

\author{
Nidhi Chahal \\ Department of Electronics \& \\ Communication Engineering \\ CEC, Landran \\ (Punjab)
}

\author{
Vinod Karar \\ Dept. of Optical Instrumentation \\ CSIR-Central Scientific \\ Instrument Organization, \\ Chandigarh
}

\author{
Manpreet Singh \\ Department of Electronics \& \\ Communication Engineering \\ CEC, Landran \\ (Punjab)
}

\begin{abstract}
This paper describes various display devices which are useful for avionics applications. It focuses the attention of cathode ray tube based displays which has distinct advantages in terms of better luminance, contrast ration and the mature level of technology. Various writing methods have been studied which are chosen depending on application, contrast level required and on the amount of information to be written in one frame. A study on handshake mechanism between the symbol generator and the display system shows that its optimization can result in optimum amount of symbol writing and removal of retraces apart from avoidance of loss of the information due to lag of driver at the display system side.
\end{abstract}

\section{Keywords}

Symbology, Head Up Display (HUD), CRT, Lissajous Pattern, Writing Speed.

\section{INTRODUCTION}

The modern aircraft cockpit is provided the pilot with ultramodern gadgets to maneuver jets flying at speed crossing sound barrier to realize the mission success efficiently. For this purpose, cockpit of an aircraft is packed with display systems viz. head-up display, helmet-mounted display, multifunctional display, standby display units, etc. [1].

There are host of graphical display reticles which play a very important role in avionics displays to provide various information about the real world parameters and aircraft to the pilot i.e. altitude, air speed, target location, RPM information, pitch ladder, heading scale, wind indicator, vertical speed, angle-of-attack bracket, energy caret, velocity vector, angle of attack, horizon bar, clock time indicator. The display computer collects information from the sources like the Inertial reference system (IRS), Air data computer (ADC), Radio altimeter, Gyros, Navigation radios and Cockpit controls. They are then translated into horizontal and vertical deflection coordinates and video blanking signal [2].

\section{DISCUSSION ON DISPLAY TECHNOLOGIES}

Various types of display technologies are available which are put into use depending on the intended applications. As a picture is worth a thousand words, display simplifies information sharing. Since its commercialization in early nineties and as recent as in early $21^{\text {st }}$ century, cathode ray tube (CRT) technology has dominated the display industry. On the other hand, new trends such as the mobile phone electronics, etc. have demanded displays that compete and outclass CRTs in certain aspects such as picture quality, power consumption and size. One of such device is liquid crystal display (LCD) which is low weight, compact in size and consumes low power. LCD based mobile phones, laptops, digital watches and electronics with miniature screen realized in smaller form factor. Other competitors in display technology are Organic LEDs, Plasma displays, Field emission displays, and Electronic paper, etc. Organic LEDs consisting of light emitting polymers emit their own light and can be realized in thin and low power consuming displays. The DLP technology uses multiple micro mirrors and can produce bright display on screens having upto 35 trillion colors. Plasma displays generates excellent quality image on very large screens. Field Emission display produces high resolution images similar to obtained on CRTs in compact size. The Electronic paper can replace print as it emulates the properties of display having paper like properties. High performance displays will ultimately drive technology evolution and this will necessitate new methods and new concepts in presentation of information. Despite of all the above facts, small flat CRT remains relevant especially for avionics application like HUD and HMD owing to its superior photometric characteristics and mature level of technology [3].

\section{OBTAINING DISPLAY ON CRT}

The CRT was invented by Karl Ferdinand Braun, the German Physicist in 1897. It is an evacuated glass envelope having an electron gun for emitting electrons. The fluorescent screen on the CRT front results in a display when accelerated and deflected electrons strike it. The deflection of electron beam and its video modulation results in display on the CRT phosphor screen [4].

The CRT accepts signal from a power-source causing negative electrode to release electrons conforming to this signal which move to the anode and the positive electrode. These electrons move in a vacuum inside the tube enclosure to the anode to strike phosphor stripes. This results in a display which may be a colour or a monochrome with shape according to the signal. The signal is required to be continuously refreshed even if its content or the color is not varying. This is required as phosphor glows for fraction of a second. The electron-beam released by the cathode sweeps horizontally across the CRT screen resulting in continuous visible picture [5].

As the discussion is on writing methods on CRT, it is worthwhile to discuss CRT types based on various principles and features. There are various ways to classify a CRT based on their color operation, display methods and functionality. A monochrome CRT has one electron gun which is made of a cathode, a negatively charged electrode and one anode 
(positively charged electrodes). The cathode emits the electrons which are attracted by the anode. The anode accelerates the electrons thus form a flow of electrons directed on the CRT screen. The deflecting magnetic field positions the electron beam from left to right and from top to bottom in the raster writing. This is generated using two electrical horizontal and vertical deflection plates responsible for horizontal and vertical flow respectively. In monochrome CRTs, green screen corresponding to "P1" phosphor screen is used though a range of phosphors like P43, P53, P1, P4, etc. are and have been used frequently for various avionics applications. Colour CRTs use alternating brightness red, green and blue phosphors. Monochrome monitors are commonly available in three colors. If the P1 phosphor is used, the screen is green monochrome [6].

The monochrome CRTs are used for avionics applications like HUD, HMD, etc. because of the fact of ease in design of optics, better eye response in green wavelength, sharper text and images than color CRTs monitors. This is possible as every pixel on monochrome CRT consists of single phosphor dot which is situated at the center of the pixel. On the other hand, pixel in case of colour CRTs consist of three phosphor dots corresponding to red, blue and green colours. No colour dot is situated at pixel center, hence lesser sharp text and image [7].

The phosphor coated color CRT consists of tiny dots set in group called as triads that contains a dye so they radiate one of the three primary colors.

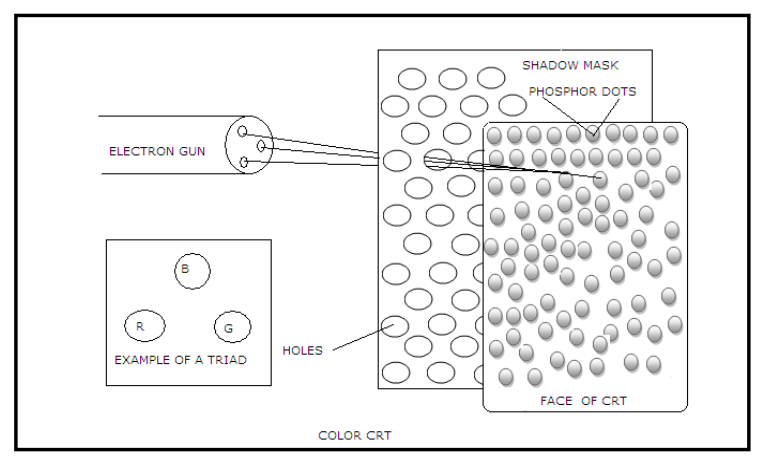

Fig 1: Color CRT

Typical grouping of triads is shown in figure 1. Resolution of CRT is generally classified by the size of the phosphor dots. The size of these dots can be as low as $0.15 \mathrm{~mm}$. These dots are the smallest addressable element of a CRT picture. They are referred to as picture elements and are abbreviated as pixels. In case of colour CRTS, three electron beams need to strike the red, blue or green colored phosphor dots. The color CRTs may employ three electron guns. Such CRTs are also referred to delta gun CRT. The electron beams navigate through a shadow mask. The shadow masks are configured in such a way that the respective colour gun strikes only that particular colour phosphor dot. In newer designs, three electron beams are combined into a one gun resulting in advantage of not requiring junction alignments which reduce the hardware required for such CRTs [8].

Another ways of classifying CRTs is based on the deflection and focusing system employed. Electromagnetic deflection method utilize magnetic field created by two pairs of deflection coils to deflect the electron beam across the CRT. Current flow develops a circular magnetic field which travels through the electron beam on its journey from the electron gun to the CRT face painted with phosphor [9].
Oscilloscopes and radar applications generally employs electrostatic deflection. The electrostatic plates control the horizontal deflection of the beam. An electrical charge is applied to these plates to direct the beam to the accurate area of the CRT. To shift the beam to the right, a positive charge is applied to the right plate to drag the beam while a negative charge is applied to the left plate to drive the electron beam to the proper position. The amount of the charge applied to the plates controls the amount of deflection [9].

Based on the display surface they are classified into flat and curved CRTs. A flat CRT has a flatter display surface than the traditional CRTs with curved display surface. The flat screen CRT results in lesser distortion at the edges as compared to the curved face CRTs [10].

CRTs are also classified based on their size into small and large CRTs. The small size CRTs having display useful surface 3 " or less finds a lot of applications like view finder of an old camcorder, Head-up Displays, Head-down displays, etc. Such CRTs have good pixel density which is nothing but dot resolution for their size. Large CRTs, on the other hand, requires contains a lot of glass. They are available in both curved as well as flat surface versions [10] [11].

\section{WRITING METHODS ON CRT TYPES OF GRAPHICS DISPLAY}

Display on CRT is obtained in similar ways the Lissajous patterns are obtained. The Lissajous curve or Lissajous figure refers to the graph of a system with following parametric equation which defines complex harmonic motion:

$$
\mathrm{X}=\mathrm{A} \sin (\mathrm{at}+\delta), \mathrm{Y}=\mathrm{B} \sin (\mathrm{bt}),
$$

The shape of Lissajous figure depends mainly on the ratio $a / b$. When $\mathrm{a} / \mathrm{b}$ is equal to 1 , resultant shape is an ellipse. When $\mathrm{A}$ $=\mathrm{B}$ and $\delta=\pi / 2$ radians, resultant shape is circle, lines result when $\delta=0$, parabola results in case of $\mathrm{a} / \mathrm{b}=2, \delta=\pi / 2$. Other ratios results in more complex curves. They are closed for the cases when $\mathrm{a} / \mathrm{b}$ is rational. The shape of these curve suggest 3 dimensional knot which project to plane as Lissajous figures [12].

Lissajous figures when $\mathrm{a}=1, \mathrm{~b}=\mathrm{N}$ where $\mathrm{N}$ is a natural number and Chebyshev polynomials of the first kind with degree $\mathrm{N}$ are defined as:

$$
\mathrm{a}=(\mathrm{N}-1) \delta / \mathrm{N} 2,
$$

The Lissajous figures with an odd natural number a, an even natural number $b, a=\delta / 2$ and $|a-b|=1$ is shown in figure 2 .
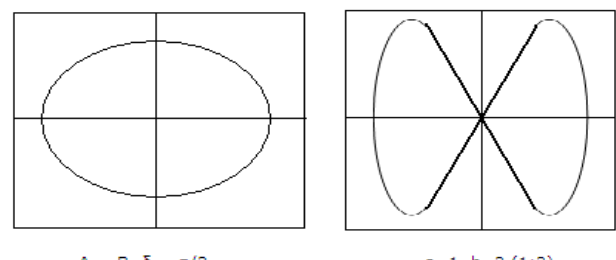

$a=1, b=2(1: 2)$

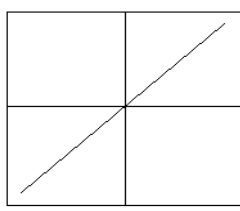

$A=B, \delta=0$

Fig 2: Lissajous Figures 
The Storage tube graphics displays uses CRTs with phosphor having longer persistence. This means that displayed character remains visible up to an hour till we erase them. The characters are drawn on the CRT by increasing the brightness of the electron beam sufficiently high causing the phosphor to be in its permanent bright storage. This image can be erased by applying the CRT tube with a definite voltage resulting in phosphor assume its dark state. Storage tube display is free from flicker and can display large number of characters and symbols. The effective resolution can be 1024 x 1024 points within an $8 \times 8$ square inch or even up to $4096 \times 4096$ points on $14 \times 14$ square inch or $18 \times 18$ square inch. However, it can't display dynamic pictures. Thus essentially it is a random scan display which facilitates drawing of vector directly within the addressable points. Thus, it plots lines and curves rather than the discrete pixels. Such display is easier to program, however, level of interactivity is lesser than refresh and raster scan display [13].

A raster CRT graphics device is a matrix of discrete cells. Each of these cells can be made bright, hence is a point plotting devices. The raster CRT graphics device uses a frame buffer which is a large and adjoining piece of computer memory. There is one memory bit for every pixel in the raster. The picture is made with the frame buffer one bit at a time. As memory bit has only two states namely 0 or 1 , a single bit plane result in black and white display. However, colour or gray levels can be assimilated into a frame buffer raster device through usage of additional bit planes. The CRT image should be transmitted at least 25 times per second to have else the CRT image will flicker [13].

The CRTs, which are meant for display of stroke form of display, uses a very short persistence phosphor. The phosphor's short persistence requires that the CRT image refreshed several times each second. The refresh rate should be greater than 30 times per second else the resultant images will flicker. Such types of display require display buffer and the display controller in addition to CRT. The display buffer embeds the required information required to paint the picture on the CRT surface while the display controller continuously refresh this information at the required rate. The factors which limit the number of vectors drawn on CRT are governed by the capacity of the display buffer and the refresh rate and speed of the display controller with speed of picture information processing also deciding on the overall performance. Thus such kind of display has similar resolution to that of a storage tube display [13].

\subsection{Symbology}

The cockpit displays installed in an aircraft cockpit provide multitude of information to the pilot by combining various sensor information on various panels like MFD, HUD, SDU computed by Mission Computer (MC) which is also referred to as Display Processor (DP). The present work deals with the symbology or display writing methods for CRT based display systems such as HUD or HMD. HUD is built around a CRT which displays the required information and data in the form of symbology, an Optical system along with partial reflector glasses which combines outside view and the symbology and project collimated symbology in pilot's front view. Flight information along with the weapon aiming information is displayed on a CRT. This information can be displayed either in composite form or stroke form. The information displayed in form of symbology refers to the approach of symbolic presentation and interpretation. They represent established sets of symbol each for which indicates definite data or information [14] [15] [16].

\subsection{Symbology Categories}

Two categories are defined for war-fighting symbology: tactical graphics and tactical symbols. Each of this can be categorized based on whether they comprise of point, line, or area objects [17].

The tactical symbols comprise of point objects which present information. This can be identified at particular location at a particular instant of time. Its constituents give information about symbol's standard identity, status, battle dimensions and mission. The shape and the size of symbols are fixed which remain same irrespective of the magnitude of the background projection, except in condition it is altered.

The tactical graphics comprise of point, line, and area entities. It is made of an icon and may have supplementary modifiers. The shape and the size of the point graphics stay stable; however, the shape and the size of the line and the area graphics are decided by drawing parameters given by the operator and the background scale upon which the graphic is placed. They are decided on the basis of necessity in battlefield planning and management, however, they cannot be presented as tactical symbols only. They can out line tasks and missions, create control measures, classify objects of concern and provide guidance.

\subsection{Writing Methods}

The symbology in day time for airborne cockpit display applications can be written in two modes depending on the application. If the application is in form of obtaining display on a MFD or SDU which has a dark background and does not get affected by the ambient brightness as much, the writing method could be a raster one. In this case, even the synthetic symbology along with the camera or the graphical display are rasterized and shown the MFD or SDU kind of displays. However, in case of see through displays like HUD or HMD, as the ambient brightness outside could be quite large, the display brightness needs to be adequately high so as to maintain the adequate contrast ratio. This will enable the pilot to view the display symbology appropriately. To achieve this, the signals are written at lesser speed so that beam stays at one point for a longer time aiding achieve more display brightness.

As discussed in above, the writing methodology and the operational principle for different CRTs vary slightly depending primarily on the type of phosphor being used. If the phosphor has long persistence, it may not be desirable for applications requiring continuous changing symbol parameters. In case of avionics applications like HUD, HMD, etc., short persistence CRTs are required which are generally made with phosphors which emit green or near green coloured light. Such phosphor characteristic will ensure that there is no tails or ghost when the display symbology changes fast. Such kind of symbology requires writing within $20 \mathrm{~ms}$ frame if the refresh rate is $50 \mathrm{~Hz}$. The useful information is followed by the blank during which the phosphor persistence ensures the presence of the display on the CRT screen. The transition between two symbols is blanked out by the video blanking signal in such a case to blank-out the retraces. In how much time a particular character or a reticle is drawn decides the brightness levels of that particular character or the reticle. All the characters or reticles can be drawn with dots, lines and arcs. They can be even drawn with dots and lines only when specific requirement of character rounding on the display symbology is not there. When alphabets or numerical or dashes or continuous dots are written, that is the time when it 
is most difficult to generate not only the horizontal and the vertical deflection signals but also the blanking signals. Slight mismatch of the deflection and the blanking signals can result in retraces or missing of the certain reticles or characters drawn. While certain delay of few microseconds can be given, but it results in unnecessary wastage of time as the signal generator will have to programmed for signal with maximum excursion. This means that even for smaller characters, the CRT based system will be required to wait till next signal is received resulting in excess DC current as well as the reduced amount of display symbology which could be drawn on the CRT surface. The alternate method of handshake could be continuous handshaking meaning thereby that the signal generator waits for the response from the CRT driven system for every horizontal and vertical deflection signal and accordingly times the blanking signal. This way, not only retraces are reduced but it also maximizes the amount of signals which can be generated during the $20 \mathrm{~ms}$ frame (see Figure 3).

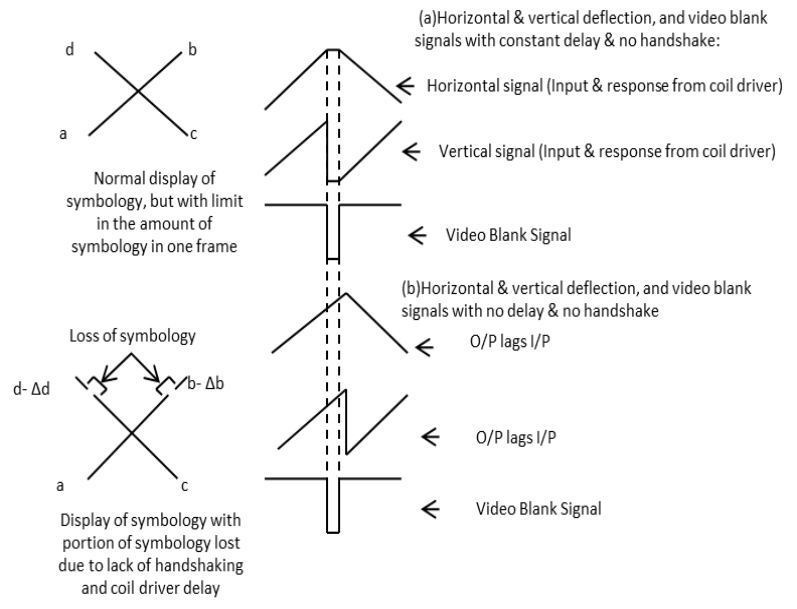

Fig 3: Horizontal \& vertical deflection, and video blank signals and display of symbology in stroke wirting method

While during the day operation, the signals need to be written slowly, the same are required to be written much faster in the night operation corresponding to the raster mixed mode of operation. This is required as the time available for stroke writing in this mode is the time available for vertical blank. This time in conventional raster scanning is $1.28 \mathrm{~ms}$ though it can be increased as per the requirement to accommodate more symbology, though it will result in raster height reduction. This requires special stroke symbol fonts in the display symbology as coil driver will not be able to follow the stroke fonts truly in such a short time. The dual scan mode operated CRT, however, can provide more time in such case well in excess of $5 \mathrm{~ms}$ which makes the symbol fonts generation and writing tasks a bit simpler. Anyway, in both the cases, that is, in conventional as well as dual raster scanning methods, the stroke symbology is written at faster speed which requires different blanking and handshaking timings making the entire writing mechanism a complex one. The amount of symbology which can be accommodated in a frame is dependent on the symbology fonts, their relative $\mathrm{dc}$ placements and full symbology composite signal amplitude.

\section{CONCLUSIONS}

Various types of display technologies were discussed in respect to their application in avionics use. The cathode ray tube display gives many advantages like wide view angle, high brightness and contrast. The methods of symbology writing are based two categories for war fighting symbology namely tactical graphics and tactical symbols. Each of this can be categorized based on whether they comprise of point, line, or area objects. Various writing methods for symbology or a CRT depend on the mode of operation, intended information to be displayed image, brightness level required and amount of symbology required to be displayed in one frame. The optimized handshaking mechanism between the symbol generator results in maximizing the symbology writing, removal of retraces and avoidance of the loss of symbology portions.

\section{REFERENCES}

[1] Saini Surender Singh, Member IAENG; Pattnaik S S; Sardana H K and Bajpai P P, "Analysis of errors and distortions in stroke form of symbology for head-up displays", Manuscript received December 29, 2011; revised January 18, 2012.

[2] A. Bowman, and J. B. Gosling, "Symbol generator for a graphical-display system", England PROC. IEEE, Vol. 122, No. 11, November1975.

[3] http://www.dolcera.com/wiki/index.php?title=LCD_Tec hnologies.

[4] http://en.wikipedia.org/wiki/Cathode_ray_tube.

[5] www.ehow .com/list_7294531_functions-cathode-ray tube.html.

[6] www.http://en.kioskea.net/contents/pc/ecran-crt.php3.

[7] www.wikipedia.org/wiki/Monochrome.

[8] www.firecontrolman.tpub.com/14102/css/14102_16.htm.

[9] www.firecontrolman.tpub.com/14102/css/14102_13.htm.

[10] www.ccs.neu.edu/home/bchafy/tiny /tiny terminal.html.

[11] http://www.howstuffworks.com/question678.htm.

[12] http:// Wikipedia// Lissajous curve.

[13] http//www.uotiq.org/dep-cs/ Computer Graphics.

[14] http://www.rto.nato.int/abstracts.asp/Automateddynamic symbology for visualization.

[15] Ertem M C, "An airborne synthetic vision system with HITS symbology using X-Plane for a head-up display", $24^{\text {th }}$ Digital avionics systems conference, Volume 2, pp 13.C.2.1-6, 2005.

[16] Burch D P, Braasch M S, "Multi-view head-up synthetic vision display system", Aerospace Conference Proceedings - IEEE, Volume 4, pp 4/1621 - 4/1630, 2003.

[17]Department of defense interface standard common war fighting symbology November 2008, superseding, MILSTD 2525B w/change, 27 March, 2007. 